\title{
Surface analytical study of hydrothermally treated zirconia ceramics
}

\author{
E. M. Moser ${ }^{1}$, B. A. Keller ${ }^{1}$, P. Lienemann ${ }^{1}$, and P. Hug ${ }^{2}$ \\ ${ }^{1}$ Swiss Federal Institute for Materials Testing and Research, CH-8600 Dubendorf, Switzerland \\ ${ }^{2}$ Institute for Inorganic Chemistry, University of Zurich, CH-8057 Zurich, Switzerland
}

\begin{abstract}
Summary. The hydrothermal instability of yttria-stabilized tetragonal zirconia polycrystals (Y-TZP) imposes serious problems on their range of applications as ceramic materials. It is well known that a generally uniform distribution of yttrium cations prevents the transformation of the metastable tetragonal into the monoclinic crystallographic modification. The induced crack propagation is widely considered to be a major source of mechanical failure in zirconia ceramics. The segregation was investigated of yttrium and other cations on the surface of hydrothermally treated ceramic samples with angle resolved XPS (X-ray photoelectron spectroscopy). The combination of this method with the high lateral resolution of SAM (scanning Auger microprobe) yields three dimensional distribution profiles of $\mathrm{Y}^{3+}, \mathrm{Al}^{3+}$ and $\mathrm{Si}^{4+}$. The relative content of the two crystallographic modifications of zirconia at the surface has been determined with XRD (X-ray diffraction). Additional Raman spectroscopic measurements partially confirm these results but suffer from quantification problems. A possible mechanism for the enrichment of yttrium in the surface layer and its promotive function for the phase transformation of zirconia is proposed. Preliminary results from experiments with temperature variable XPS measurements also indicate the formation of either yttrium- or zirconium hydroxide which are both suspected to contribute to crack propagation along the grain boundaries.
\end{abstract}

\section{Introduction}

Yttria-stabilized zirconia ceramics are known as Y-TZP (yttria tetragonal zirconia polycrystals). The extraordinary strength and toughness of this ceramic material are due to the metastable tetragonal grains. These can transform to the monoclinic form when external stress is applied. However the tetragonal grains may transform spontaneously to the monoclinic modification on exposure to humid conditions in the temperature range of 100 to $500^{\circ} \mathrm{C}$, leading to serious degradation of the material [1]. Several mechanisms have been proposed for this phase transformation. Sato and coworkers [2] have suggested a slow crack growth mechan-

Correspondence to: E.M. Moser ism to be responsible for the low temperature degradation. Their mechanism is based on the assumption that water reacts with the $\mathrm{Zr}-\mathrm{O}-\mathrm{Zr}$ bonds at the tip of a crack, with the consequence that $\mathrm{Zr}-\mathrm{OH}$ is formed. Yoshimura [3], suggested that chemisorption of water induces an $\mathrm{OH}^{-}$migration from the surface to the bulk, resulting in nucleation defects. A third model has been postulated by Lange et al. [4]. These authors proposed that the reaction between water and yttria at the ceramic surface leads to the formation of $\alpha-Y(O H)_{3}$ islands. Spontaneous transformation to the monoclinic modification will take place after yttrium depletion in exposed grains through this process. After reaching a critical loss of yttrium the monoclinic structure growth will follow a classical nucleation and growth mechanism. Some support for this model is given by Wang and Stevens [5], who describe the phase transformation as a similar process. According to their observations the ageing behaviour depends on the degree of homogeneity, with regard to the distribution of yttrium in zirconia. The presence of small numbers of nucleation elements, however, only generates a compressive layer instead of forming microcracks. This effect has been called surface toughening [5].

Hithero only a few surface analytical studies have been carried out on the phenomenon of low temperature degradation of Y-TZP ceramics. Winnubst and Burggraaf [6] confirmed Lange's model with AES spectroscopy, observing a strong yttrium segregation to the surface after water ageing treatments of $\mathrm{Y}-\mathrm{TZP}$ ceramics with varying yttria contents. In a UHV system attached to a high pressure cell Axelsson et al. [7] heated monoclinic zirconium oxide in water. This process caused a segregation of silicon, which is always present in zirconia ceramics, to the surface. XPS measurement undertaken by Lintula et al. [8] revealed hydroxide groups at the surface of aged Y-TZP material but not inside the transformed layer.

The intention of this present work was to pursue the events taking place at the surface of hydrothermally treated Y-TZP ceramics with surface sensitive techniques, such as XPS, SAM/AES and Raman spectroscopy. XRD was used to determine the monoclinic phase content of the material. Further details about XRD studies are given in Stevens et al. [9] and in reference [1]. Since numerous papers have been published dealing with the optimization of ceramic material with respect to mechanical properties this work was limited to study the surface distribution of the cations. 


\section{Experimental}

Yttria-doped zirconia ceramics Y-TZP containing $3.0 \mathrm{~mol} \%$ (5.2 wt $\%$ ) yttrium oxide were obtained from Metoxit AG, Oxidkeramik, CH-8240 Thayngen. Samples containing 3.0 mol\% yttria and trace amounts of silica and alumina was kindly provided by Prof. L.J. Gauckler (ETH, Zurich) and his group.

Hydrothermal treatment (distilled water, $230^{\circ} \mathrm{C}, 5$ bar) has been carried out in an autoclave over periods of 1,3 and $4 \mathrm{~h}$, respectively. Polished (diamond paste of $3 \mu \mathrm{m}$ ) as well as finely ground or recrystallized $\left(1300^{\circ} \mathrm{C}\right.$ for $\left.3 \mathrm{~h}\right)$ samples were investigated. The chemical composition of the material was established by ICP-MS (inductively coupled plasma mass spectroscopy) and is given in Table 1. All samples have been ultrasonically cleaned in ethanol prior to characterization by XRD, XPS and Raman spectroscopy (before and after hydrothermal treatment).

Photoelectron spectroscopy measurements were performed with a Perkin Elmer PHI 5400 small spot ESCA apparatus equipped with a channel plate detector and a $\mathrm{Mg}$ $\mathrm{X}$-ray source operated at $400 \mathrm{~W}(15 \mathrm{kV} ; 26,7 \mathrm{~mA})$. The pass energy of the analyzer was set to $35.75 \mathrm{eV}$, resulting in a full width at half-maximum (FWHM) value of $1.0 \mathrm{eV}$ for the $\operatorname{Ag}\left(3 \mathrm{~d}_{5 / 2}\right)$ line. A residual pressure lower than $9 \cdot 10^{-10} \mathrm{mbar}$ has been maintained in the analytical chamber during the measurements. The $\mathrm{Zr}(3 \mathrm{~d})$ line at $182.2 \mathrm{eV}$ was used as a reference to correct for sample charging [10]. For quantification the $\mathrm{Zr}(3 \mathrm{~d})-, \mathrm{Y}(3 \mathrm{~d})-, \mathrm{Si}(2 \mathrm{p})-, \mathrm{Al}(2 \mathrm{p})$ - and $\mathrm{O}(1 \mathrm{~s})$ - core level regions have been integrated. Due to unknown sensitivity factors of the oxides the corresponding values for the elements were used to calculate the number of ions present at the surface [11].

The elemental distribution has also been studied with a Perkin Elmer PHI Model 4300 SAM spectrometer operating with a primary beam energy of $3 \mathrm{kV}$ and an electron beam current of $0.01 \mu \mathrm{A}$. The samples were heated up to $450^{\circ} \mathrm{C}$ to enhance the ion conductivity of the material while its thermal stability is still maintained [12]. Electron probe for microanalysis (EPMA) and scanning electron microscopy (SEM) were used to investigate the microstructure and precipitations in polished and thermally etched samples.
Raman spectra have been recorded with a Biorad FTS spectrometer unit equipped with a $\mathrm{Nd}$ : YAG laser (CVI C-95) as excitation source. The power level of the NIR radiation $(1064 \mathrm{~nm})$ has been kept constant at $450 \mathrm{~mW}$. Generally a resolution of $2 \mathrm{~cm}^{-1}$ has been found to be sufficient to resolve the bands originating from the monoclinic and tetragonal crystal modifications. Due to serious limitations in sensitivity and cut-off of the laser fundamental line, it was not possible to quantify the results from the Raman data alone. Therefore additional measurements with X-ray diffraction (XRD) were necessary to monitor the extent of the phase transition from the thermodynamically metastable tetragonal to the stable monoclinic modification.

XRD measurements have been performed on a Siemens diffractometer (D-500) using $\mathrm{CuK}_{\mathrm{r}}$ radiation. The semiquantitative evaluation of the content of transformed monoclinic phase has been determined by the decrease in the integrated tetragonal diffraction line [111]. The existence of a cubic zirconia modification has not been observed in these experiments.

\section{Results}

\section{$X P S$ and $S A M / A E S$}

The experimentally obtained abundance of different ions and oxygen as a function of $\sin \Theta$ (with $\Theta$ being the take off angle), are displayed in Figs. 1 to 4 and in Table 2. The con-

Table 1. Chemical composition of the polished Y-TZP samples as numbered according to the system used in Table 2

\begin{tabular}{lllrrrrr}
\hline Sample & $\begin{array}{l}\mathrm{Y}_{2} \mathrm{O}_{3} \\
{[\mathrm{wt} \%]}\end{array}$ & \multicolumn{2}{c}{$\mathrm{Y}[\mathrm{ct} \%]$} & $\begin{array}{l}\mathrm{SiO}_{2} \\
{[\mathrm{wt} \%]}\end{array}$ & $\mathrm{Si}[\mathrm{ct} \%]$ & $\begin{array}{l}\mathrm{Al}_{2} \mathrm{O}_{3} \\
{[\mathrm{wt} \%]}\end{array}$ & $\mathrm{Al}$ [ct\%] \\
\hline TZP 0 & 5.18 & 5.82 & $<0.01$ & $<0.06$ & $<0.01$ & $<0.06$ \\
TZP 1 & 5.18 & 5.82 & 0.03 & 0.09 & $<0.01$ & $<0.06$ \\
TZP 2 & 5.15 & 5.79 & 0.06 & 0.12 & 0.05 & 0.12 \\
TZP 3 & 5.17 & 5.76 & 0.33 & 0.68 & 0.22 & 0.56 \\
TZP 4 & 5.08 & 5.67 & 0.78 & 1.54 & 0.45 & 1.13 \\
\hline
\end{tabular}

Table 2. Experimental concentration and enrichment factors of cations in the investigated ceramic samples

\begin{tabular}{|c|c|c|c|c|c|c|c|c|}
\hline Sample ${ }^{a}$ & $\mathrm{Y} / \mathrm{Zr}\left(15^{\circ}\right)$ & $\mathrm{Y} / \mathrm{Zr}\left(43^{\circ}\right)$ & $\operatorname{Si}\left(15^{\circ}\right)^{b}$ & $\operatorname{Si}\left(43^{\circ}\right)^{b}$ & $\operatorname{Al}\left(15^{\circ}\right)^{b}$ & $\operatorname{Al}\left(43^{\circ}\right)^{\mathrm{b}}$ & $f\left(Y, 15^{\circ}\right)^{c}$ & $\mathrm{~m}^{\mathrm{d}}$ \\
\hline TZP 0.0 & 5.2 & 5.8 & 17.7 & 4.5 & $<1.5$ & $<1.5$ & 0.8 & -- \\
\hline TZP 0.1 & 10.3 & 8.6 & 27.3 & 3.0 & - & 2.8 & 1.7 & 80 \\
\hline TZP 0.2 & 8.1 & 6.0 & 5.2 & 3.1 & 5.8 & 2.4 & 1.3 & 80 \\
\hline TZP 0.3 & 7.3 & 6.5 & 4.8 & 3.4 & 2.0 & $<1.5$ & 1.2 & 80 \\
\hline TZP 1.1 & 9.8 & 7.5 & 19.4 & 5.8 & $<1.5$ & $<1.5$ & 1.6 & $<5$ \\
\hline TZP 1.3 & 10.8 & 10.0 & 8.6 & 3.0 & -- & 3.6 & 1.8 & $<5$ \\
\hline TZP 2.1 & 9.2 & 8.9 & 9.6 & 5.9 & 6.1 & 2.4 & 1.5 & 40 \\
\hline TZP 2.3 & 14.6 & 10.0 & 6.8 & 3.2 & 6.8 & 3.8 & 2.4 & 70 \\
\hline TZP 3.2 & 10.8 & 8.7 & 9.2 & 4.0 & 5.3 & 3.0 & 1.8 & 10 \\
\hline TZP 4.2 & 7.7 & 7.0 & 18.1 & 4.7 & 10.8 & 7.4 & 1.2 & 50 \\
\hline
\end{tabular}

${ }^{\mathrm{a}}$ Time of hydrothermal treatment: .0: untreated; $.1: 1 \mathrm{~h} ; .2: 3 \mathrm{~h} ; .3: 4 \mathrm{~h}$, resp

${ }^{\mathrm{b}}$ Concentration [cation \%] at take off angle $\Theta=15^{\circ}$ and $43^{\circ}$, resp

${ }^{\mathrm{c}}$ Enrichment factor of yttrium: the ratio $\mathrm{Y} / \mathrm{Zr}$ is divided by the bulk concentration $\mathrm{Y} / \mathrm{Zr}=6.18 \%$

${ }^{d}$ Monoclinic phase content [\%] determined with XRD 


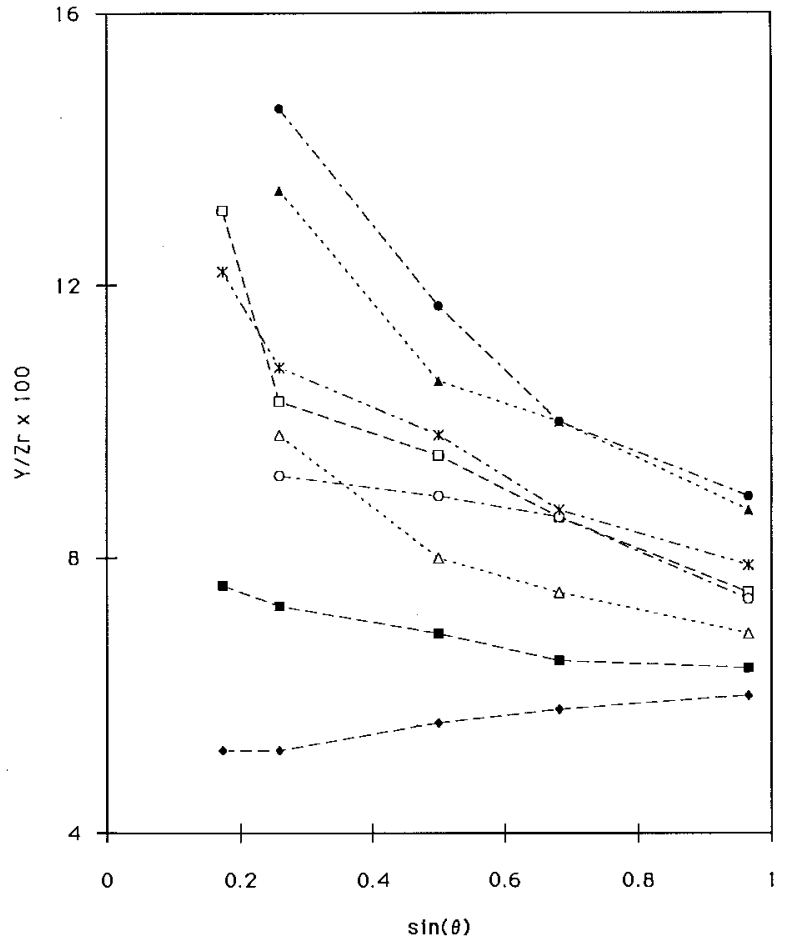

Fig. 1. Relative concentration of yttrium ions as a function of $\sin (\Theta)$. $\ldots-\ldots$ TZP $0.0 ;--\square--$ TZP $0.1 ;--\square--T Z P 0.3 ; \ldots . \triangle \ldots$ TZP

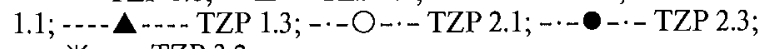
$-\cdots-\nVdash-\cdot \cdot-$ TZP 3.2

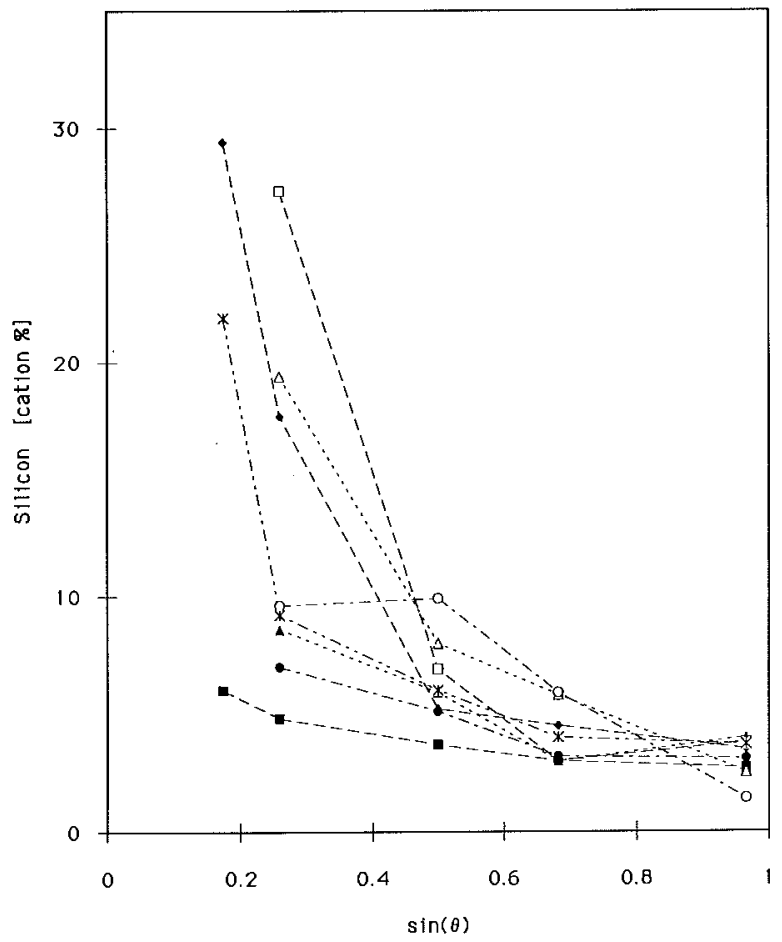

Fig. 2. Concentration of silicon ions as a function of $\sin (\Theta)$. - TZP $0.0 ;--\square--$ TZP $0.1 ;--\square--T Z P ~ 0.3 ;-.-\triangle-. .-$ TZP 1.1 ; --- $\triangle$-..- TZP $1.3 ;-\cdot-O-\cdot-$ TZP $2.1 ;-\cdot-\bullet-\cdot$ TZP $2.3 ;-\cdots-X-\cdots$ TZP 3.2

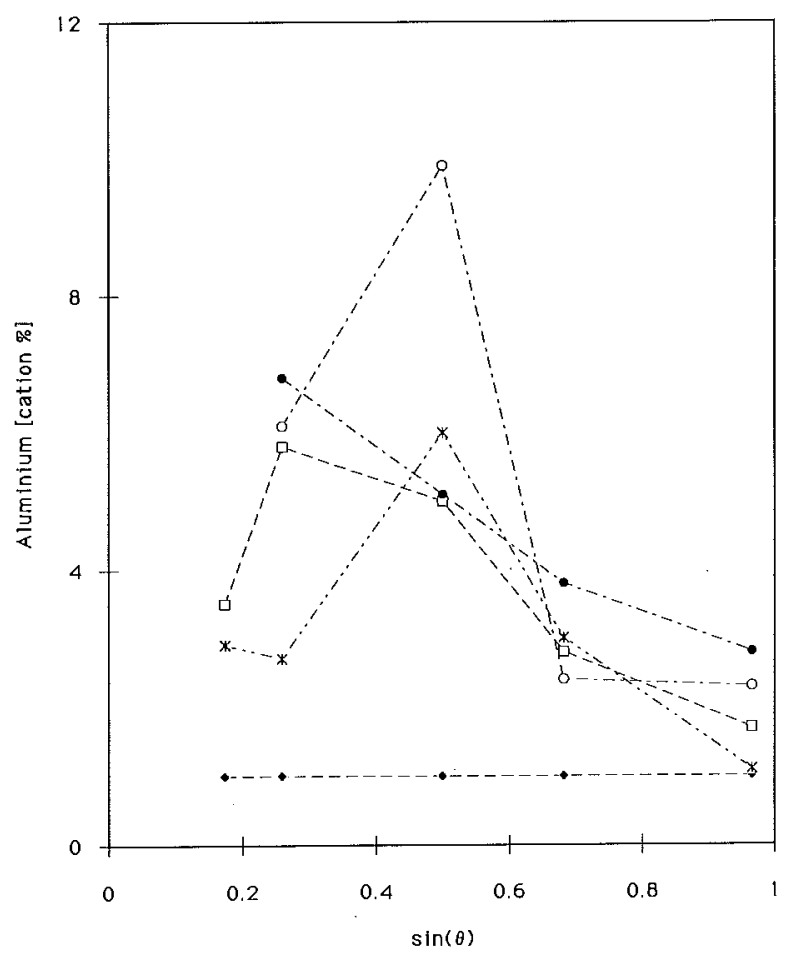

Fig. 3. Concentration of aluminium ions as a function of $\sin (\Theta)$. --•-- TZP $0.0 ;--\square--$ TZP $0.2 ;-\cdot-\mathrm{O}-\cdot-$ TZP $2.1 ;-\cdot-\bullet-\cdot-$ TZP $2.3 ;---X--$ TZP 3.2

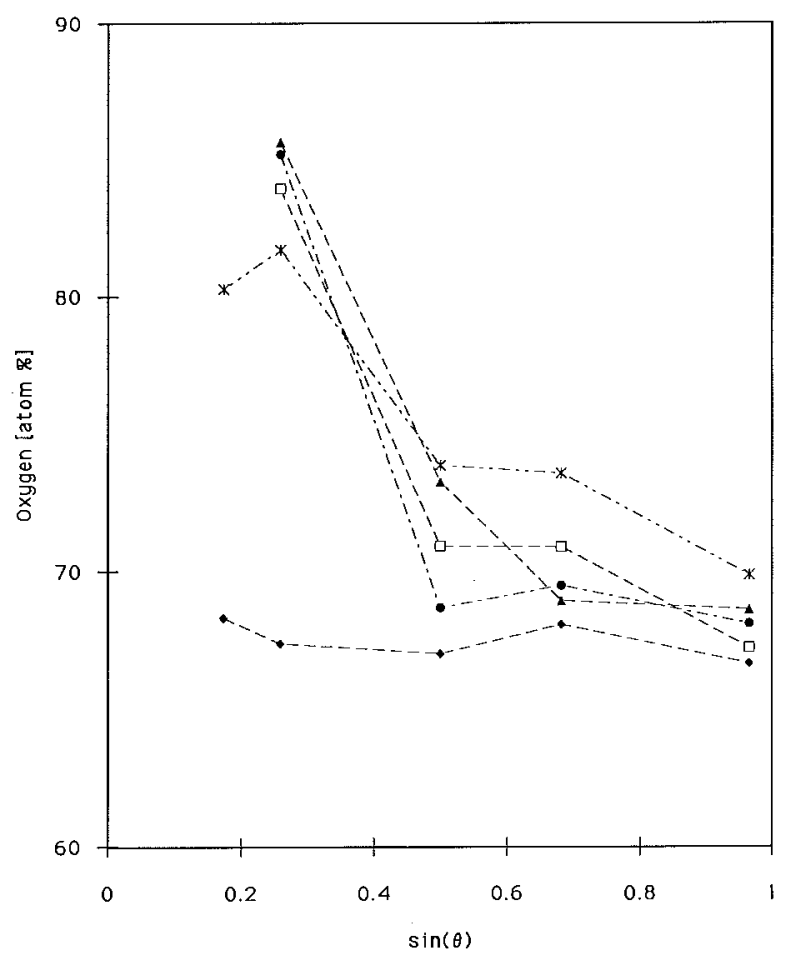

Fig. 4. Concentration of oxygen as a function of $\sin (\Theta)$. -- -- TZP $0.0 ;--\square--$ TZP $0.1 ;-. .-\Delta$-..- TZP $1.3 ;-\cdot-\bullet-\cdot-$ TZP $2.3 ;-\cdots-X-\cdots-$ TZP 3.2 
centration ratio of yttrium to zirconium (multiplied by a factor of 100) together with an enrichment factor for yttrium $\mathrm{f}\left(\mathrm{Y}, \Theta=15^{\circ}\right)$ are given in Table 2 . According to these experimental data, the enrichment factor does not exhibit a clear dependence on the ageing time.

The depth profiles of the relative concentrations of yttrium to zirconium as shown in Fig. 1 indicate a significant enrichment in the case where the phase transformation from the tetragonal to the monoclinic crystal modification has just started (TZP 1 to TZP 4). With increasing time of hydrothermal treatment this feature passes through a maximum value and then gradually disappears again (samples TZP 0.1 to TZP 0.3 ).

The silicon concentration, as a function of the information depth, is displayed in Fig. 2. In the outermost layers of all polished samples a high amount (up to $30 \mathrm{ct} \%, \Theta=15^{\circ}$ ) of silicon ions $\left(\mathrm{Si}^{4+}\right)$ has been found experimentally. However, after hydrothermal treatment of several hours, this behaviour is no longer evident and a slight decrease rather than an enrichment of the silicon ion concentration can be observed in the spectra taken at $\Theta=75^{\circ}$, which corresponds to an information depth of about 6-8 nm.

In all hydrothermally treated samples containing various small ( $<0.01-0.45$ wt. \%) amounts of alumina, a very strong migration of the aluminium ions has been observed (Fig. 3). The enrichment is most prominent in the first few subsurface layers. A correlation of the aluminium enrichment and the monoclinic phase content is shown in Table 2.

A comparison of the polished and hydrothermally treated samples in Fig. 4 indicates that a large amount of water has been incorporated into the top few surface layers. Temperature variable XPS measurements show that a peak located at $533.3 \pm 0.3 \mathrm{eV}$ in the $\mathrm{O}(1 \mathrm{~s})$ core level region disappears at about $250^{\circ} \mathrm{C}$. This binding energy is characteristic for water. The coincidence with the maximum reaction temperature estimated for the Y-TZP material with water is striking [1].

$O(1 \mathrm{~s})$ spectra and their differences are depicted in Fig. 5 with the oxygen peak located at a binding energy of 530.2 $\pm 0.2 \mathrm{eV}$, which is assigned to the oxide of zirconium. The oxygen species removed by heating the sample up to $500^{\circ} \mathrm{C}$ has an $\mathrm{O}(1 \mathrm{~s})$ binding energy of $531.9 \pm 0.3 \mathrm{eV}$ which is typical for hydroxides. This temperature has been estimated as the upper limit for low temperature degradation. It was therefore concluded that the oxygen species observed is either yttrium or zirconium hydroxide. Unfortunately the limited resolution of the spectrometer and the overlapping positions of the hydroxide peak for yttrium and zirconium prevent a distinction between the two. However atomic percentage numbers obtained from the difference spectrum clearly show that as well as yttrium hydroxide, a considerable amount of zirconium hydroxide must also be present.

SAM element mapping of several polished and aged ceramic surfaces resulted in homogeneous distributions for the various ions within the instrumental lateral resolution of $0.2 \mu \mathrm{m}$. However the relatively high amount of silica and alumina in the sample TZP 4 resulted in the formation of zircon silicate $\left(\mathrm{ZrSiO}_{4}\right)$ and small $\mathrm{Al}_{2} \mathrm{O}_{3}$-precipitations on the surface. These results have been confirmed with EPMA measurements. Zircon silicate could not be detected after hydrothermal treatment.

A SEM image of the aged sample TZP 4.2 and the corresponding yttrium Auger image (SAM) are given in Figs. 6 and 7, respectively. Enhanced yttrium and oxygen concen-

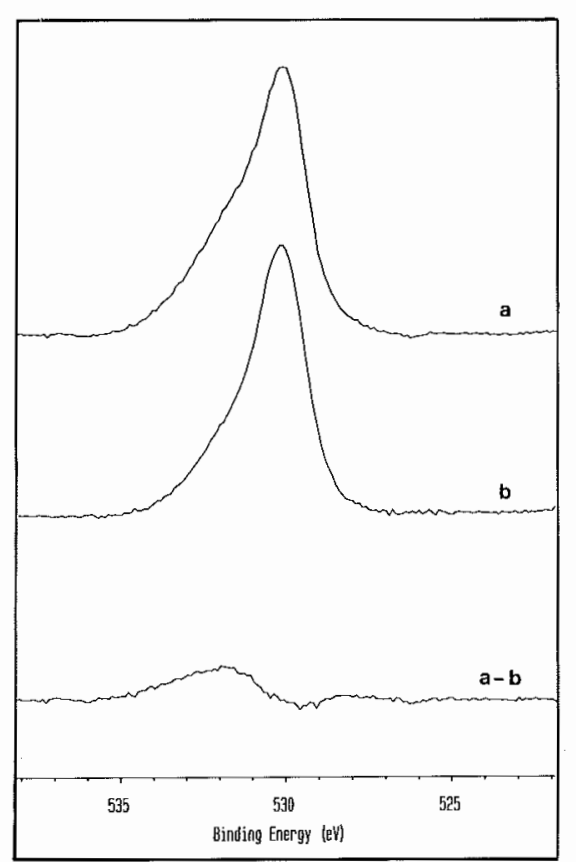

Fig. 5. XPS spectra of sample TZP 0.3 taken at $\Theta=90^{\circ}$

a) $\mathrm{O}(\mathrm{s})$ core level region at $250^{\circ} \mathrm{C}$

b) $\mathrm{O}(1 \mathrm{~s})$ core level region recorded at room temperature after heating the sample to $500^{\circ} \mathrm{C}$.

a-b) $O(1 s)$ difference spectrum

trations are observed within the bright regions in the upper left-hand corner of the picture. Therefore it was concluded that the observed yttrium/oxygen compound is most probably a precipitation of yttrium hydroxide. These findings are in qualitative agreement with the work of Lange et al. [4] who detected $\alpha-\mathrm{Y}(\mathrm{OH})_{3}$ in a TEM experiment.

\section{Raman spectroscopy and XRD}

The ratios between monoclinic and tetragonal crystal modifications have been measured with XRD and are shown in Table 2. Raman measurements partly confirmed these findings, but the relatively small penetration depth of the NIR radiation into the optically opaque ceramic material yields misleading figures for the bulk phase transition. It has, however, been possible to identify both crystal modifications at the surface of several samples. In the spectral region between 100 and $300 \mathrm{~cm}^{-1}$ the monoclinic doublet at 181 and $192 \mathrm{~cm}^{-1}$, and the tetragonal bands at 148 and $264 \mathrm{~cm}^{-1}$ are well separated. These Raman lines are only relatively weak in these spectra compared to the literature [13] due to the radiative transition properties of the cut-off filter for the laser fundamental line. Therefore the monoclinic band around $380 \mathrm{~cm}^{-1}$ was used for experimental verification of phase transitions. Raman spectra were obtained from polished and powdered samples with an enhanced sensitivity for the latter. Care has to be taken in cases where samples had to be ground because mechanical stress can induce phase transition. Figure 8 shows the Raman spectra of samples TZP 0 after different ageing times. 

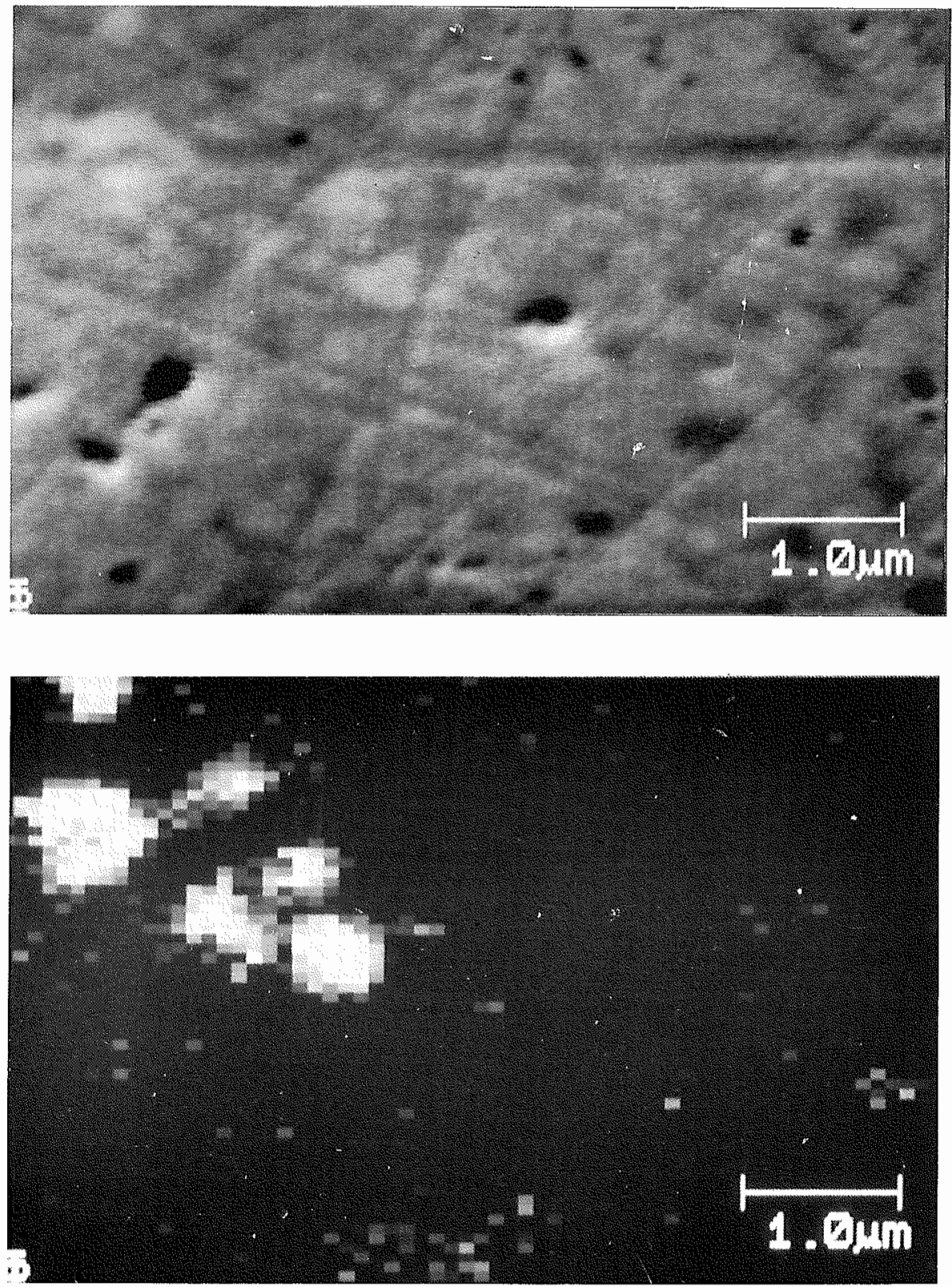

Fig. 7. Yttrium Auger image (SAM) for the same surface area as shown in Fig. 6
Fig. 6. SEM image of sample TZP 4.2 at a magnification of $20 \mathrm{kx}$

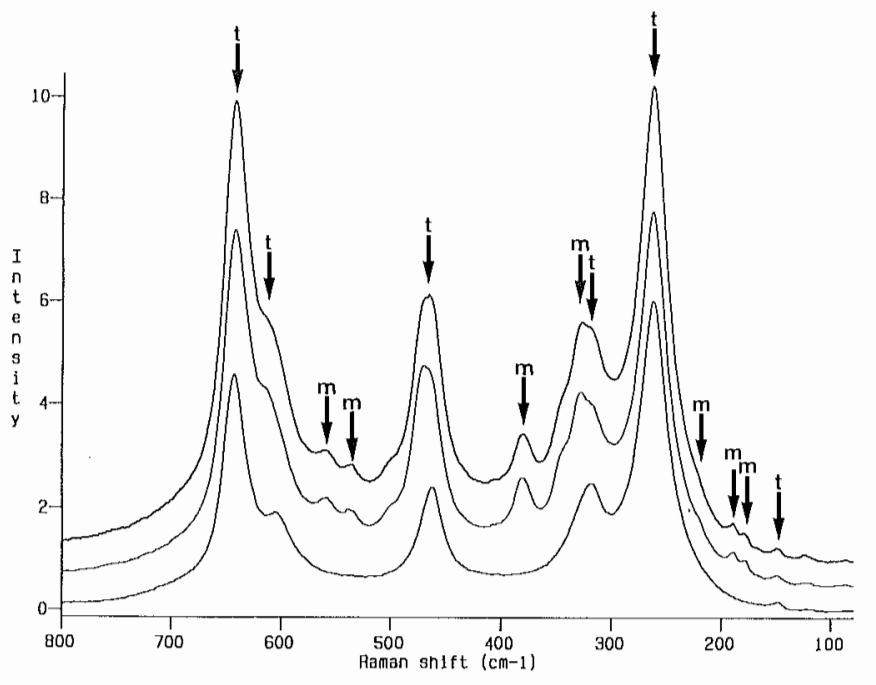

Fig. 8. Raman spectra of TZP samples recorded with $400 \mathrm{~mW}$ excitation power and $2 \mathrm{~cm}^{-1}$

TZP 0.0 (a), TZP 0.2 (b) and TZP 0.3 (c)

$\mathrm{m}=$ monoclinic bands, $\mathrm{t}=$ tetragonal bands 


\section{Discussion}

The experimental results presented here indicate that the enrichment of yttrium ions at the surface of various samples may be correlated with the amount of phase transition to the monoclinic modification. Small differences in the chemical composition strongly affect the microstructure and the segregation of the yttrium ions and as a consequence the ageing behaviour. Measurements of XPS depth profiles of thermally treated and water aged Y-TZP surfaces, which showed enrichment factors of up to 2.7 for yttrium, are in qualitative agreement with the work of Winnubst and Burggraaf [6]. A thorough discussion of the segregation of yttrium ions to the grain boundaries and to the surfaces, together with the correlation of the chemical composition and microstructure with this phenomenon will be published in a subsequent paper. The present results also strongly support the concept proposed by Lange et al. [4] that the formation of $\mathrm{Y}(\mathrm{OH})_{3}$ islands may trigger the start of phase transformation through a decrease in the amount of yttria below a critical value in certain predisposed (size and composition) TZP grains.

The strong enrichment of silicon ions at the outermost surface layers indicates a different behaviour of the silicon ions compared to that of yttrium and aluminium. The silicon XPS data cannot be correlated directly with the monoclinic phase content. However the decreasing enrichment of silicon after prolonged hydrothermal treatment indicates a dissolution of surface silica and zircon silicate in water. Axelsson et al. [7] heated monoclinic zircon oxide without stabilizers in a water atmosphere of $1070 \mathrm{~K}$ and 16 Torr and observed a migration of silicon to the surface. The authors proposed the formation of the thermodynamically favoured zircon silicate after a subsequent oxidation at $1070 \mathrm{~K}$.

\section{Conclusions}

The segregation of various cations in yttria-stabilized zirconia ceramics (Y-TZP) has been investigated with XPS, SAM/AES, Raman spectroscopy and XRD. It has been shown that yttrium ions are enriched in the top monolayers during the beginning of the hydrothermal treatment whereas silicon ions tend to be removed from the outer surface by an unknown mechanism. Depth profiles of aluminium ions showed a strong enrichment in the subsurface layers of the material.

During the progress of ageing the yttrium concentration gradually drops to a value to close to the initial bulk value in the sample; this leads to the assumption that segregation of yttrium within the ceramic lattice may initially trigger the observed phase transformation. Although these results do not conclusively end the controversy in the literature about the initial steps in this mechanism, the simultaneous occurrence of yttrium segregation and the local enrichments of yttrium/oxygen compounds at some sample surfaces indicates that the proposed mechanism may play a role in it. The major conclusion from the experiments presented in this work is a strong support for the fact that the homogeneity of the yttrium ion distribution within the ceramic material finally determines the resistance against low temperature degradation.

Acknowledgements. The authors wish to thank Dr. P. Richner for ICPMS measurements, P. Boll for EPMA investigations and C. Schenk for recording SEM pictures. Special thanks are due to B. Michel for providing us with the Y-TZP samples from her thesis work.

\section{Refereences}

1. Lilley E (1989) Corrosion and corrosive degradation of ceramics. Anaheim, CA (references therein)

2. Sato T, Shimada M (1985) J Am Ceram Soc 68:356-59

3. Yoshimura M, Noma T, Kawabata K, Somija S (1987) J Mater Sci Lett 6:465-67

4. Lange FF, Duniop GL, Davis BI (1586) J Am Ceram Soc 69: $237-40$

5. Wang J, Stevens R (1989) Ceram Int 15:15-21

6. Winnubst AJA, Burggraaf AJ (1988) Advances in ceramics, science and technology of zirconia III, Vol 24A

7. Axelsson KO, Keck K-E, Kasemo B (1986) App1 Surf Sci 25 : 217-230 (references therein)

8. Lintula P, Lepistö TT, Reuhkala P, Mäntylä T (1989) Euro Ceram $\mathrm{I}: 1542-1546$

9. Wang J, Stevens R (1989) J Mat Sci Lett $8: 1195-1198$

10. Hughes AE, Sexton BA (1989) J Mater Sci $24: 1057$

11. Wagner CD, Riggs WM, Davis LE, Moulder JF, Mullenberg GE (1979) Handbook of X-ray photoelectron spectroscopy. Perkin Elmer Corporation, Eden Prairic

12. Hamminger R (1988) SIA 12:519-526

13. Clark DR, Adar F (1982) J Am Ceram Soc 65 :284-288 